\title{
Design of a Dual-Band Sectoral Antenna for Hiperlan2 Application Using Double Layers of Metallic Electromagnetic Band Gap (M-EBG) Materials as a Superstrate
}

\author{
M. Hajj, ${ }^{1}$ R. Chantalat, ${ }^{2}$ and B. Jecko' \\ ${ }^{1}$ Faculté des Sciences et Techniques, XLIM - CNRS UMR 6172, 123 Avenue Albert Thomas, 87060 Limoges, France \\ ${ }^{2}$ CISTEME, Ester Technopole, 87069 Limoges Cedex, France
}

Correspondence should be addressed to M. Hajj, mohamad.hajj@xlim.fr

Received 1 February 2009; Accepted 5 May 2009

Recommended by Lei Zhu

A novel design of a sectoral antenna that utilizes a double layer Metallic Electromagnetic Band Gap (M-EBG) as a superstrate for dual band directivity enhancement is presented in this paper. We obtain the different operating frequencies by adjusting the distance of the lower M-EBG layer from printed patch antenna and also the height between upper and lower M-EBG layers. This antenna operates according to a sectoral radiation pattern form presenting a half power beamwidth of at least $60^{\circ}$. The proposed structure presents more than $17 \mathrm{~dB}$ directivity enhancement at $5.25 \mathrm{GHz}$ and $5.65 \mathrm{GHz}$ as compared to those of a patch antenna with $9 \mathrm{~dB}$ directivity. The principle is explained and applied to a Hiperlan 2 antenna.

Copyright () 2009 M. Hajj et al. This is an open access article distributed under the Creative Commons Attribution License, which permits unrestricted use, distribution, and reproduction in any medium, provided the original work is properly cited.

\section{Introduction}

The electromagnetic Band Gap (EBG) materials are periodic structures in where the wave propagation for certain frequency bands and certain incidence angles is prohibited $[1,2]$.

The insertion of a defect within the crystal EBG periodicity can create an allowed band inside the forbidden band. By exciting the defect structure at the allowed band frequency it was proven that it is possible to design antennas with interesting performances.

The one-dimensional radiation is ensured by the insertion of a ground plane in the middle of the defect [2-4].

Recently, these materials have been used to design different types of antennas with directive [3-6], omnidirectional $[7,8]$, or sectoral radiation patterns $[9,10]$, where the MEBG is constituted by a layer of metallic rods.

Our objective was to obtain a dual-band metallic EBG antenna able to radiate according to a sectoral form pattern presenting a half power beamwidth of at least $60^{\circ}$ in one of the planes $[9,10]$.
In recent years, metamaterials based on Electromagnetic Band Gap (EBG) structures using Frequency Selective Surface (FSS) have been widely investigated in the antennas domain for dual-band frequency and the bandwidth enhancement [10-14].

In [10-14] a single and multilayer FSS with metallic strip elements have been proposed as superstrate layers over patch antenna to increase the patch directivity in single- and multioperating frequency bands.

In this paper, we propose an M-EBG consisting of a twolayered Metallic EBG superstrate with same periodicities for dual-band directivity enhancement.

Because the superstrates made by dielectric rod layers and plates are difficult to fabricate in practice and a specific dielectric constant is not always available, M-EBG can be good candidates as alternatives to dielectric rod layers or plates.

The structure proposed is able to generate two adjacent bands which are needed by telecommunications standards such as UMTS or Hiperlan2, these bands can be used to cover both uplink and downlink for the same application. 


\section{Characteristics and Design of M-EBG Superstrate Layers}

The usual method to investigate the characteristics of the structure is examining both superstrate layer and its image on the ground plane using image theory [15].

The M-EBG structure is infinitely periodic in $x, y$ directions and finite in $z$ direction (Figure 1). Therefore, its properties are characterized using periodic boundary condition for each unit cell.

To obtain the transmission coefficients of the structure in Figure 1, it is illuminated from the bottom in the $z$ direction by a linearly polarized plane wave.

Figure 1(a) shows one cell of M-EBG layer consisting of infinite numbers of metallic rods with $a=2 \mathrm{~mm}$, the periodicity $p=10 \mathrm{~mm}$. The M-EBG structure is printed on a dielectric layer whose thickness $b=5 \mathrm{~mm}$ and the dielectric constant is 1.45 .

Depending on the number of operating frequencies, the superstrate above the patch antenna can have one layer (Figure 1(a)) or more (Figure 1(b)).

The two-layered M-EBG structures are the same in terms of dimension, periodicity, and thickness.

For dual-band directivity enhancement, we introduce 2 defects by inserting $2 \mathrm{M}$-EBG layers with the same properties above the ground plane (Figure 1(b)).

The defects generated by the ground plane and the layers of metallic rods will be referred to herein as Defect 1 with $D 1=30.25 \mathrm{~mm}$ and Defect 2 with $D 2=28.75 \mathrm{~mm}$, respectively (Figure $1(\mathrm{~b})$ ).

The proposed structure acts as a resonator at two different frequencies. The first one is approximately determined by the distance between the ground plane and the lower MEBG layer ( $f 1$ ) (Defect 1), while the other is governed by the distance between upper and lower M-EBG layers ( $f 2)$ (Defect 2) (Figure 1(b)).

Figure 2 shows the transmission coefficient of one MEBG layer, two M-EBG layer cases versus frequency. The results present several interesting properties. The black curve shows two different defect modes exist in the bandgap, and they are useful for dual-band operation (Figure 2).

For example, the defect frequencies appear at $4.16 \mathrm{GHz}$ $(f 1)$ and $4.65 \mathrm{GHz}(f 2)$ with the upper M-EBG layer, while there exists only one defect frequency at $4.25 \mathrm{GHz}$ without Defect 2.

We can observe several transmission bands in the band gap. In this case especially, we will use two modes at $f 1=$ 4.16 and $f 2=4.65 \mathrm{GHz}$ for the radiation of our antenna.

Figure 3 shows the electric field distributions for different defect modes (mode 1-4).

From this plot, we see that the first and fourth modes have maxima at the location of the ground plane, while the second and third modes are zero there. Hence, only the latter two modes can be available in the M-EBG superstrate, and not the others, because only in these modes tangential electric fields on symmetrical plane (Ground Plane) are zero [15].
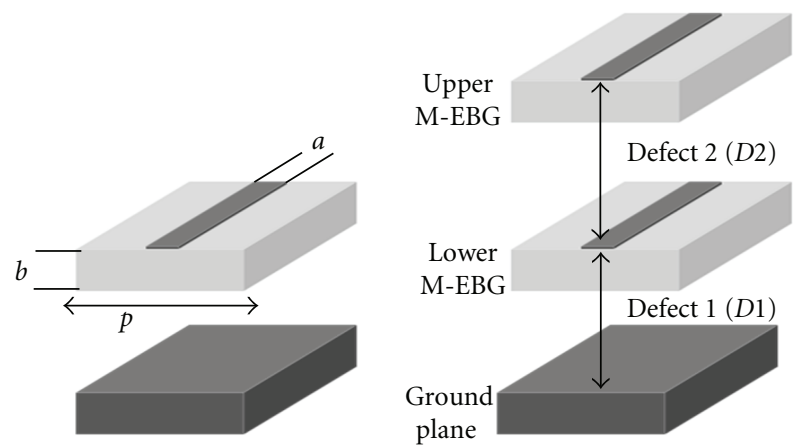



(a)



Figure 1: (a) Unit cell geometry of the one layer M-EBG. (b) Unit cell geometry with two layer M-EBG.

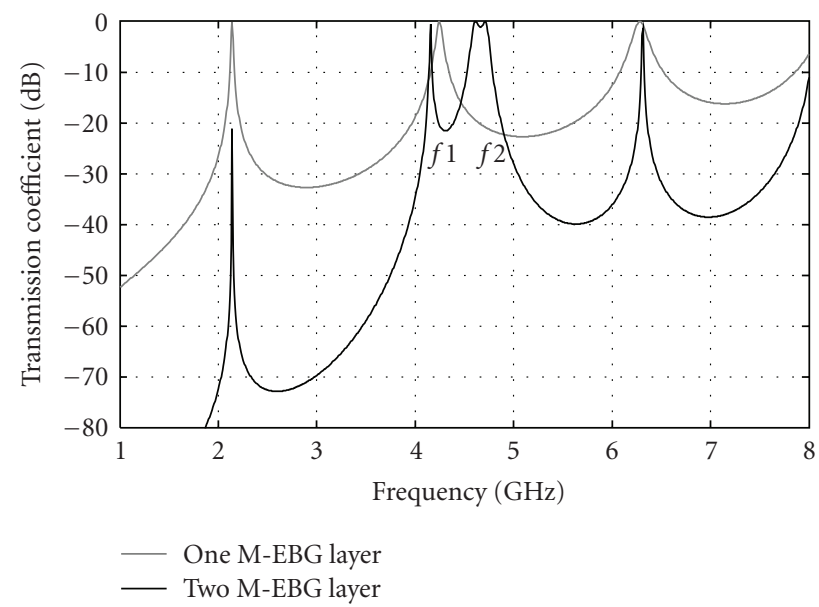

FIGURE 2: Transmission coefficient characteristics with and without the upper M-EBG layer.

Thus, the insertion of a ground plane at this location does not modify the properties of the material. Nevertheless, it has the advantage of reducing the height of the structure by half.

The variations of the directive M-EBG antenna directivity, with and without Defect 2, are shown in Figure 4.

To obtain a maximum directivity greater than $20 \mathrm{~dB}$ at the two bands, an M-EBG superstrate comprised of 36 metallic rods is designed above a printed patch antenna.

The lower M-EBG superstrate makes a directivity enhancement at one frequency band, we stack another MEBG layer above the existing single-band one, in order to achieve directivity enhancement in a second band (Figure 4). 


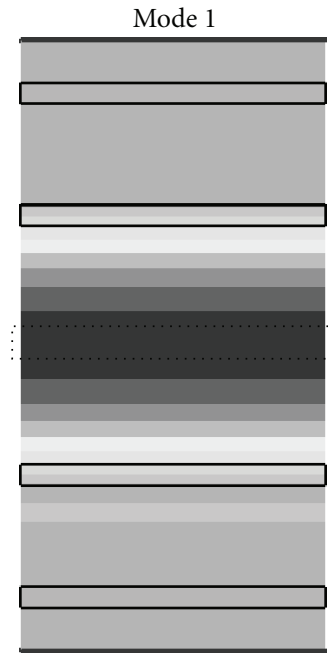

$f=2.14 \mathrm{GHz}$

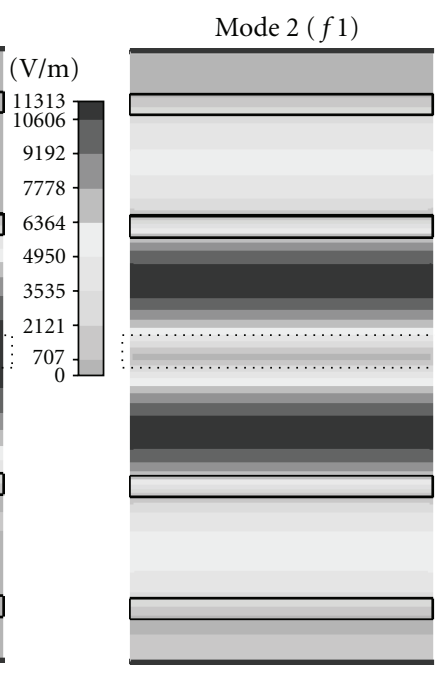

$f=4.16 \mathrm{GHz}$

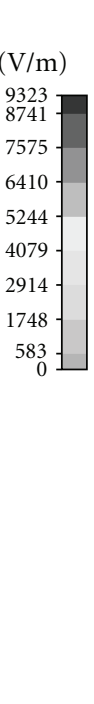

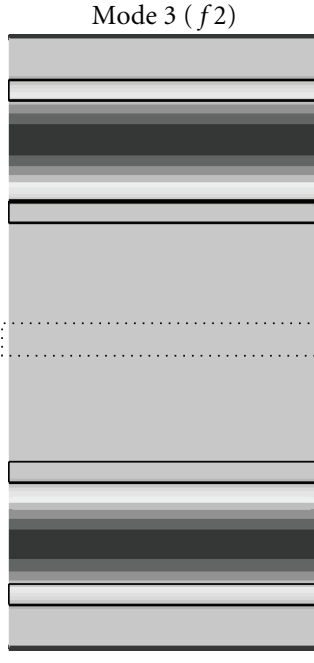

$f=4.65 \mathrm{GHz}$


FIGURE 3: Electric field distributions of different modes in the M-EBG structure.

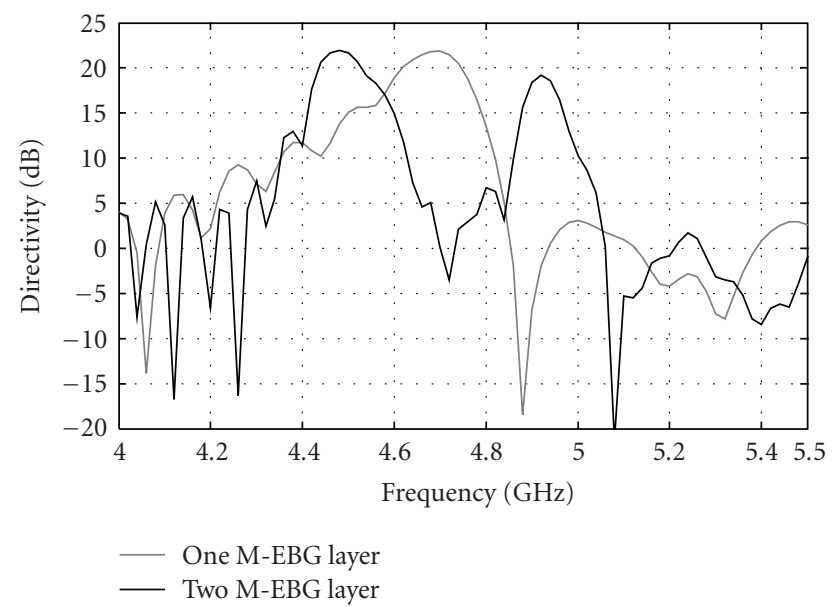

FIGURE 4: Comparison of the simulated directivity with and without the upper M-EBG layer.

Simulation results show that the operating frequencies 4.46 GHz and 4.92 GHz (Figure 4) are closely associated with the transmission peaks of unit cell that are $4.16 \mathrm{GHz}$ and 4.65 GHz (Figure 2).

\section{Dual-Band M-EBG Sectoral Antenna Design}

This work aims at the design of a dual-band sectoral antenna useful for Hiperlan2 Network applications, which operates at two frequency bands, namely, 5.15-5.35 GHz as an uplink and 5.47-5.725 GHz as a downlink, respectively, with $17 \mathrm{~dB}$ directivity and a half power beamwidth of $60^{\circ}$ in the horizontal plane.

This antenna uses one-dimensional M-EBG structures in TM polarization (the rods are parallel to the E field) as a superstrate reflective walls. The Dual-Band M-EBG antenna is composed of three principal parts (Figure 5).

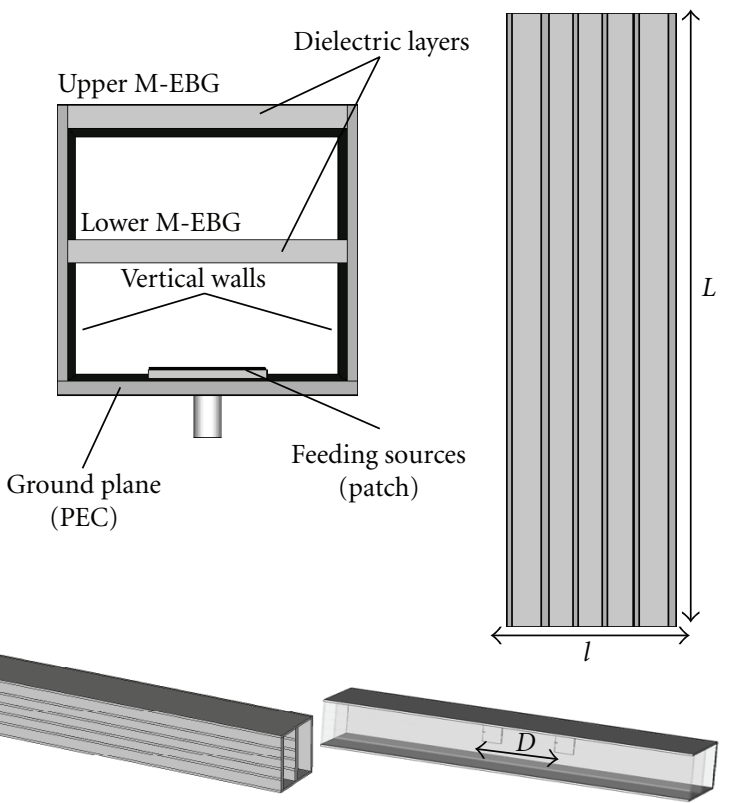

Figure 5: Front, Top, and 3D views of the Dual-Band Sectoral MEBG antenna.

(1) The ground plane where the feeding system (2 patches) is located.

(2) The two-layered M-EBG structures as a superstrate made by periodic metallic elements.

(3) The vertical walls to ensure the sectoral functionality of the antenna.

A sectoral radiation pattern can be obtained by using a rectangular radiating aperture. This can be achieved with an EBG resonator antenna, provided that the energy propagation is impaired in one direction of the azimuth plane. 




- Dual-band M-EBG sectoral antenna - 2 patches only

FIgURe 6: Directivity evolution for the two cases.

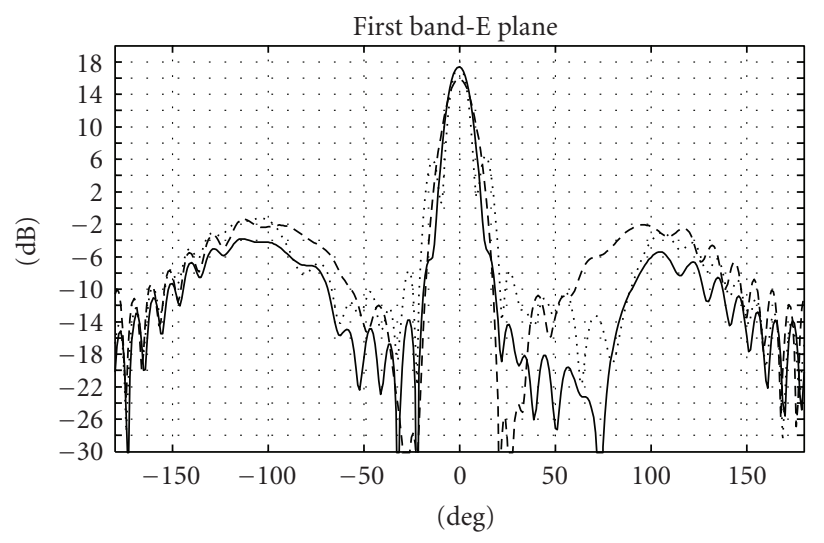

(a)



(b)

FIGURE 7: Radiation patterns: (a) E plane, $f=5.15,5.25,5.32 \mathrm{GHz}$ (b) H plane, $f=5.15,5.25,5.32 \mathrm{GHz}$.

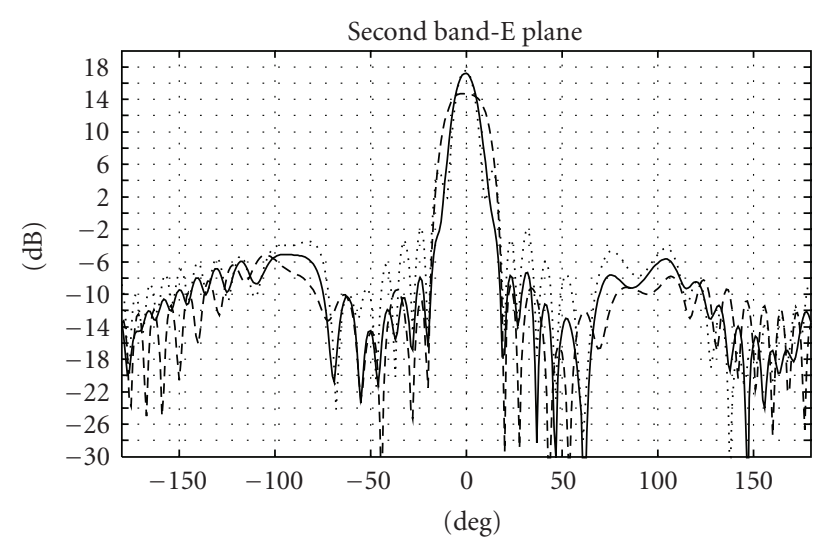

(a)

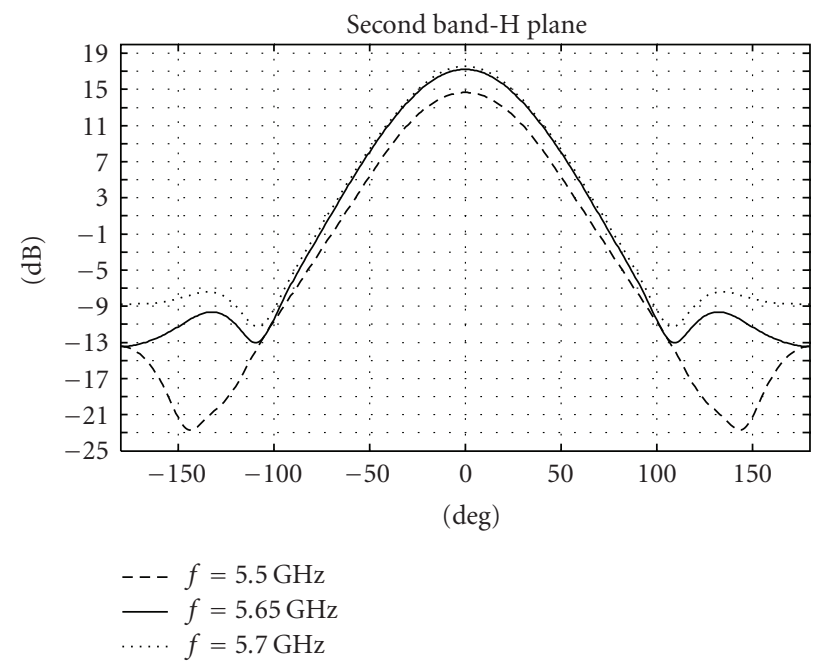

(b)

FIGURE 8: Radiation patterns: (a) E plane, $f=5.5,5.65,5.7 \mathrm{GHz}$ (b) H plane, $f=5.5,5.65,5.7 \mathrm{GHz}$.

The use of vertical walls into the cavity (Figure 5) to limit the energy propagation results in the desired effect. Those will limit the repartition of energy, leading to the desired spot.

The wall confines the field and preserves it inside the cavity by limiting its spreading out in the horizontal plane. Consequently the radiant spot extends and takes an elliptic form $[9,10]$.

The dual-band sectoral M-EBG antenna made up of 4 metallic rods in order to adjust the quality factor at each band (directivity greater than $17 \mathrm{~dB}$ ) and to assure sectoral radiation (sectoral aperture of $60^{\circ}$ ).

The feeding system presents two patches spaced out by $D=55 \mathrm{~mm}$ correspond to $\lambda_{0}$. In our design we use a square patch with $20 \mathrm{~mm}$ side. This patch is printed on a thin substrate layer (thickness $2 \mathrm{~mm}, \varepsilon_{r}=1.45$ ).

The simulated ground plane has the same size as the structure, that is, $L=375 \mathrm{~mm} * L=56 \mathrm{~mm}$. 


\section{Simulated Results of the Final Structure}

The antenna structure has been simulated with CST Microwave Studio, a Finite Integration Technique (FIT) based software.

The simulation results are shown in Figure 6 which represents the directivity versus frequency.

As expected, there are two separate frequency bands with maximum directivities greater than $17 \mathrm{~dB}$ (see Figure 6) compared to that of two patches only case (12 dB max).

In the frequency bands (5.15-5.35), (5.47-5.725) GHz, the directivity varies between $14 \mathrm{~dB}$ and $17.2 \mathrm{~dB}$.

We observe that the two frequency bands obtained with the directive M-EBG antenna (see Figure 4) shift to upper frequencies (see Figure 6) as the vertical walls are introduced into the cavity.

The operating frequencies match exactly the frequencies used for the Hiperlan2 network system.

The E-plane and H-plane radiation patterns at the two radiation bands frequencies are shown in Figures 7 and 8, respectively.

In the E plane the radiation is directive; on the other hand, the radiation pattern in the horizontal plane presents an interesting sectoral aperture of $60^{\circ}$.

The patterns have low side lobes, so we can estimate that the structure volume is well dimensioned.

\section{Conclusion}

This manuscript proposes an original application of M-EBG materials to build dual-band sectoral antennas with several advantages.

The study of EBG materials showed us that their electromagnetic properties allow designing high gain antennas design.

Two highly reflective M-EBG layers are used as the superstrate for a resonant cavity fed by a patch array to create a dual frequency band.

We showed that the planar structures can be adapted to obtain structures whose radiation is sectoral $>60^{\circ}$ in azimuth plane and directive in the vertical plane.

\section{References}

[1] E. Yablonovitch, "Photonic band-gap structures," Journal of Optical Society of America B, vol. 10, no. 2, 1993.

[2] G. V. Trentini, "Partially reflecting sheet arrays," IEEE Transactions on Antenna and Propagation, vol. 4, no. 4, pp. 666-671, 1956.

[3] M. Thevenot, C. Cheype, A. Reineix, and B. Jecko, "Directive photonic-bandgap antennas," IEEE Transactions on Microwave Theory and Techniques, vol. 47, no. 11, pp. 2115-2122, 1999.

[4] C. Cheype, C. Serier, M. Thevenot, T. Monediere, A. Reineix, and B. Jecko, "An electromagnetic bandgap resonator antenna," IEEE Transactions on Antennas and Propagation, vol. 50, no. 9, pp. 1285-1290, 2002.

[5] D. R. Jackson and N. G. Alexopoulos, "Gain enhancement methods for printed circuit antennas," IEEE Transactions on Antennas and Propagation, vol. 33, no. 9, pp. 976-987, 1985.
[6] M. Qiu and S. He, "High directivity patch antenna with both photonic bandgap substrate and photonic bangap cover," Microwave and Optical Technology Letters, vol. 30, no. 1, pp. 41-44, 2001.

[7] E. Pointereau, H. Chreim, P. Dufrane, and B. Jecko, "Omnidirectional cylindrical electromagnetic bandgap antenna with dual polarization," IEEE Transactions on Antenna and Propagation, vol. 6, pp. 450-452, 2007.

[8] H. Chreim, E. Pointereau, P. Dufrane, and B. Jecko, "Omnidirectional electromagnetic band gap antenna for base station applications," IEEE Transactions on Antenna and Propagation, vol. 6, pp. 499-502, 2007.

[9] M. Hajj, E. Rodes, D. Serhal, et al., "Metallic EBG sectoral antenna for base stations," in Proceedings of the 19th International Conference on Applied Electromagnetics and Communications (ICECom '07), pp. 1-4, Dubrovnik, Croatia, September 2007.

[10] M. Hajj, D. Serhal, E. Rodes, and T. Monédière, "Dual-band metallic FSS-EBG sectoral antenna," in Proceedings of IEEE International Symposium on Antennas and Propagation and USNC/URSI National Radio Science Meeting (APSURSI '08), pp. 1-4, San Diego, Calif, USA, July 2008.

[11] E. Rodes, M. Diblanc, E. Arnaud, T. Monédière, and B. Jecko, "Dual-band EBG resonator antenna using a single-layer FSS," IEEE Antennas and Wireless Propagation Letters, vol. 6, pp. 368-371, 2007.

[12] Y. J. Lee, J. Yeo, R. Mittra, and W. S. Park, "Design of a frequency selective surface (FSS) type superstrate for dualband directivity enhancement of microstrip patch antennas," in Proceedings of IEEE Antennas and Propagation Society International Symposium and USNC/URSI Meeting, vol. 3, pp. 2-5, Washington, DC, USA, July 2005.

[13] Y. J. Lee, J. Yeo, R. Mittra, and W. S. Park, "Thin frequency selective surface (FSS) superstrate with different periodicities for dual-band directivity enhancement," in Proceedings of IEEE International Workshop on Antenna Technology: Small Antennas and Novel Metamaterials (IWAT '05), pp. 375-378, Singapore, March 2005.

[14] A. P. Feresidis and J. C. Vardaxoglou, "High gain planar antenna using optimised partially reflective surfaces," IEE Proceedings: Microwaves, Antennas and Propagation, vol. 148, no. 6, pp. 345-350, 2001.

[15] Y. J. Lee, J. Yeo, R. Mittra, and W. S. Park, "Application of electromagnetic bandgap (EBG) superstrates with controllable defects for a class of patch antennas as spatial angular filters," IEEE Transactions on Antennas and Propagation, vol. 53, no. 1, pp. 224-235, 2005. 

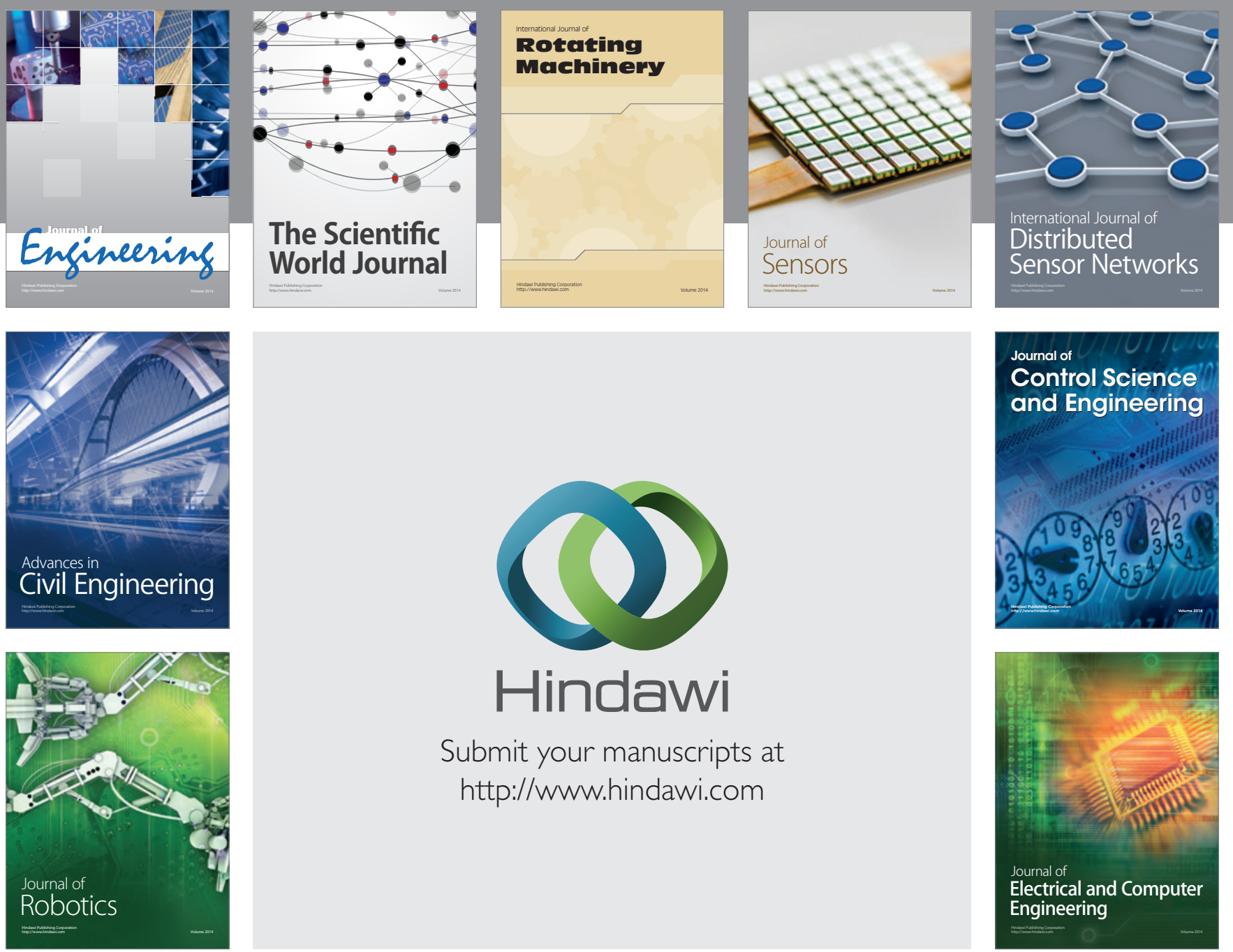

Submit your manuscripts at

http://www.hindawi.com
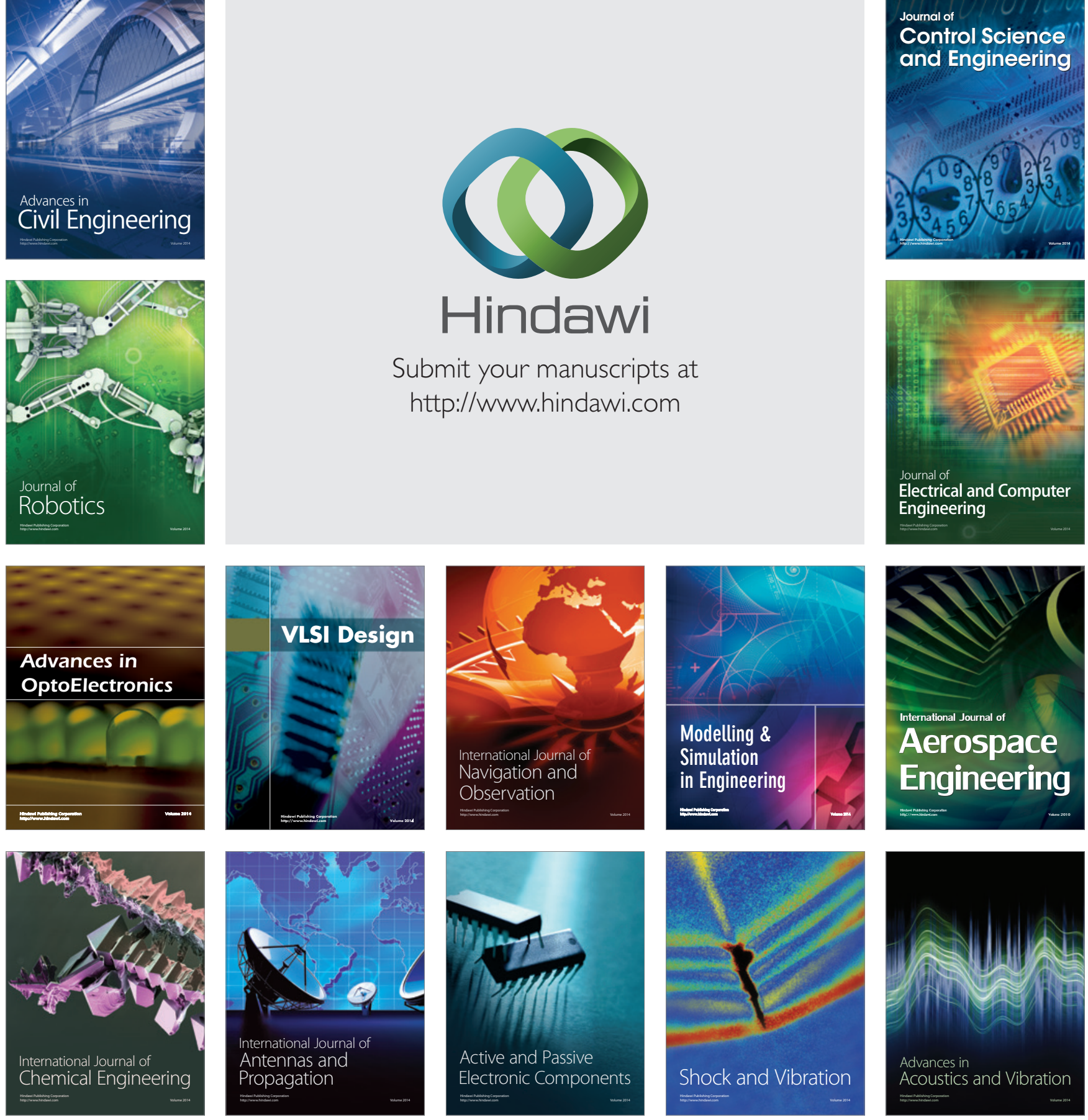